\title{
Molecular phylogenetic analyses reveal two new synonyms of Xanthoria parietina
}

\author{
Andrei Tsurykau ${ }^{1,2^{*}}$, Pavel Bely ${ }^{3} \&$ Ulf Arup ${ }^{4}$
}

\section{Article info}

Received: 20 Mar. 2020

Revision received: 1 Oct. 2020

Accepted: 2 Oct. 2020

Published: 29 Dec. 2020

Associate Editor

Adam Flakus

\begin{abstract}
Molecular analyses of the recently described Xanthoria polessica collected from the type locality and of the Australian species $X$. coomae were used to determine whether these taxa are distinct species or fall within the wide phenotypic variation of the wellknown and cosmopolitan $X$. parietina. Our results clearly indicate that both taxa should be considered as synonyms of $X$. parietina since the infraspecific morphological range is accommodated by the observed variation in the thallus and lobe size, their color, position of apothecia, the shape of ascospores, and width of ascospore septum.
\end{abstract}

Key words: taxonomy, phylogeny, ITS, Teloschistaceae, lichens

\section{Introduction}

The well-known lichen genus Xanthoria is currently represented by at least nine species, namely $X$. aureola, $X$. calcicola, X. ibizaensis, X. mediterranea, X. monofoliosa, X. parietina, X. resendei, X. steineri and X. stiligera (Arup et al. 2013; Kondratyuk et al. 2020), but there are many unresolved names and the taxonomy of the genus is in need of molecular evaluation. Recently, two new species were described in this genus, namely $X$. polessica and X. juniperina (Kondratyuk et al. 2013), but their descriptions were based only on morphological characters and were never supported by molecular data. Phylogenetic analyses by Arup et al. (2013) indicated that the Australian species $X$. coomae (Kondratyuk et al. 2007) probably belongs to $X$. parietina .

In our study, we focused on the species $X$. polessica and $X$. coomae, the former described by Kondratyuk et al. (2013) based on an old herbarium specimen from 1967 stored in MSK herbarium in Minsk. In addition, 29 specimens from rather different areas of Belarus, Russia and Ukraine have been cited giving an idea of the widespread occurrence of this species. Fieldwork conducted by the second author (PB) at the well-preserved type locality yielded fresh material of $X$. polessica, allowing us to

\footnotetext{
${ }^{1}$ Department of Biology, Francisk Skorina Gomel State University, Sovetskaja 104, BY-246019 Gomel, Belarus

${ }^{2}$ Department of Ecology, Botany and Nature Protection, Institute of Natural Sciences, Samara National Research University, Moskovskoye shosse 34, RU-443086 Samara, Russia

${ }^{3}$ Laboratory of Ecological Physiology of Plants, Central Botanical Garden of the National Academy of Sciences of the Republic of Belarus, Surganova 2B, BY-220012 Minsk, Belarus

${ }^{4}$ Biological Museum, Lund University, Box 117, SE-22100 Lund, Sweden

* Corresponding author e-mail: tsurykau@gmail.com
}

obtain ribosomal DNA sequences (ITS) of the species. The performed phylogenetic analyses indicate that $X$. polessica is conspecific with $X$. parietina. In the case of $X$. coomae, the previous suggestion by Arup et al. (2013) that the species belongs to $X$. parietina has been confirmed in this study. Therefore, we propose $X$. polessica and $X$. coomae to be synonymous with $X$. parietina. The high morphological variability of $X$. polessica is discussed.

\section{Material and methods}

\section{Morphological examination}

Morphology and anatomy were studied using Nikon SMZ745 and Nikon Eclipse 80i microscopes (Tokyo, Japan). Handmade sections of ascomata were studied in water and $5 \% \mathrm{KOH}(\mathrm{K})$. Measurements of ascospores size and length/width ratio $(1 / \mathrm{w})$ are given as (minimum-) X-SD $-\mathrm{X}+\mathrm{SD}$ (-maximum), where $\mathrm{X}$ represents the arithmetic mean and SD the corresponding standard deviation, followed by the number of measurements (n).

DNA amplification, sequencing, alignment and phylogenetic analysis

Two ITS sequences were obtained from the two collections of $X$. polessica using direct PCR following the procedure described in detail in Arup et al. (2015). Primers for amplification were ITS1F (Gardes \& Bruns 1993) and ITS4 (White et al. 1990). An alignment was created to include the two newly generated ITS sequences of $X$. polessica together with 34 sequences downloaded from GenBank (Table 1) of X. aureola, X. calcicola, X. coomae, $X$. ectaneoides (according to the name in Genbank, but the relationship to $X$. aureola is unclear), $X$. mediterranea, 
Table 1. Location, collector and GenBank accession numbers of sequences of species of Xanthoria used in the analyses. Specimens in bold were newly produced for this study.

\begin{tabular}{|c|c|c|}
\hline Species & Location and collector & $\begin{array}{c}\text { GenBank Accession } \\
\text { Number }\end{array}$ \\
\hline Dufourea flammea & South Africa, Feuerer and Thell 60488a (HBG) & KC179357 \\
\hline X. aureola & Sweden, Lindblom X188 (priv.herb.) & AY438276 \\
\hline$X$. aureola & United Kingdom, 2002, Coppins s. n. (E) & AY438275 \\
\hline X. calcicola & Sweden, Arup L97372, (LD) & AF353944 \\
\hline X. calcicola & Sweden, Lindblom 1199CL (BG) & AY438282 \\
\hline X. coomae & Australia, Kondratyuk 204116 (KW) & EU681291 \\
\hline X. coomae & Australia, Kondratyuk 20455 (KW) & EU681292 \\
\hline$X$. coomae (holotype) & Australia, Kondratyuk 20494 (CANB) & KC179410 \\
\hline$X$. ectaneoides & France, Unknown s.n. (G) & AJ320131 \\
\hline$X$. mediterranea & Italy, Honegger s.n. (G) & AM408410 \\
\hline X. monofoliosa & South Africa, Thell 00196, (LD) & EU681293 \\
\hline$X$. parietina & Italy, Arup L97905 (LD) & AF353943 \\
\hline$X$. parietina & Switzerland, Unknown 98 (??) & AJ320118 \\
\hline$X$. parietina & Russia, Honegger 213+4 (Z+XT) & AM292826 \\
\hline$X$. parietina & Sweden, Lindblom \& Blom L111 (BG) & AY438310 \\
\hline$X$. parietina & Sweden, Lindblom 1/1 (BG) & AY926499 \\
\hline$X$. parietina & Norway, Lindblom 1/3 (BG) & AY926500 \\
\hline$X$. parietina & Norway, Lindblom 1/15 (BG) & AY926502 \\
\hline$X$. parietina & Norway, Lindblom 2/36 (BG) & AY926504 \\
\hline$X$. parietina & Norway, Lindblom 2/37 (BG) & AY926505 \\
\hline$X$. parietina & Norway, Lindblom 2/52 (BG) & AY926509 \\
\hline$X$. parietina & Norway, Lindblom 11/340 (BG) & AY926513 \\
\hline$X$. parietina & United Kingdom, Unknown s.n. (KEW) & FR799309 \\
\hline$X$. parietina & United Kingdom, Unknown s.n. (KEW) & KJ027704 \\
\hline$X$. parietina & Germany?, Unknown s.n. (??) & KF590005 \\
\hline$X$. parietina & Germany?, Unknown s.n. (??) & KF590014 \\
\hline$X$. parietina & Unknown, Grande \& Singh s.n. (??) & KJ027703 \\
\hline$X$. parietina & Unknown, Unknown s.n. (??) & KY379230 \\
\hline$X$. parietina & Norway, Arnstein Lye O-L-206852 (O) & MK811702 \\
\hline$X$. parietina & Norway, Bendiksby O-L-196081 (O) & MK812134 \\
\hline$X$. parietina & Norway, Timdal O-L-195782 (O) & MK812349 \\
\hline$X$. parietina & Denmark, Søchting s.n. (C) & KC179411 \\
\hline$X$. parietina & Chile, Frödén 1620 (L) & KC179412 \\
\hline X. polessica & Belarus, Bely s.n. (LD, GSU, MSKH) & MT928332 \\
\hline X. polessica & Belarus, Bely s.n. (LD, GSU, MSKH) & MT928333 \\
\hline$X$. resendei & Unknown origin, Lich 13259 (BCN) & AF101285 \\
\hline$X$. cf. stiligera & Spain, Moestrup s.n. (C) & KC179409 \\
\hline
\end{tabular}

X. monofoliosa, X. parietina, X. resendei and X. cf. stiligera. Dufourea flammea was used to root the tree (Arup et al. 2013). The 37 sequences were aligned using MAFFT ver. 7.450 (Katoh \& Standley 2013; Katoh et al. 2002) as implemented in Geneious 11.0.5 and then improved manually, including a total of 523 positions, of which 53 were phylogenetically informative.

A suitable model of molecular evolution was selected using the Bayesian Information Criterion (BIC) as implemented in jModeltest ver. 2.1.4 (Guindon \& Gascuel 2003; Darriba et al. 2012) to evaluate only the 24 models available in MrBayes 3.2.4 (Ronquist et al. 2012). The $\mathrm{SYM}+\mathrm{G}$ model was found to be optimal. Bayesian tree inference was carried out using Markov chain Monte Carlo (MCMC) as implemented in MrBayes 3.2.4. The number of discrete categories used to approximate the gamma distribution was set to 4 . The following priors were used: beta $(1,1)$ on the transition-transversion rate, fixed on the state frequencies, uniform $(0 \cdot 001,200)$ for the gamma shape parameter, and all trees a priori equally likely. The prior on branch lengths for the analyses was set to an exponential with a mean of $1 / 10$. Three parallel runs were performed, each with 6 chains, 5 of which were incrementally heated with a temperature of $0 \cdot 10$. Analyses were diagnosed every 100,000 generations and automatically halted when convergence was reached. Convergence was defined as a standard deviation of splits (with frequency $b \geq 0 \cdot 1$ ) between runs below $0 \cdot 01$. Every 1,000 th tree was sampled and the first $50 \%$ of runs were removed as burn-in. FigTree 1.4 (http://tree.bio.ed.ac.uk/ software/figtree/) and Adobe Illustrator CS4 were used to construct and illustrate a phylogenetic consensus tree.

\section{Results}

A 50\% majority rule consensus tree from the post burn-in trees is presented in Figure 1. The phylogenetic tree shows a monophyletic and well delimited Xanthoria parietina 


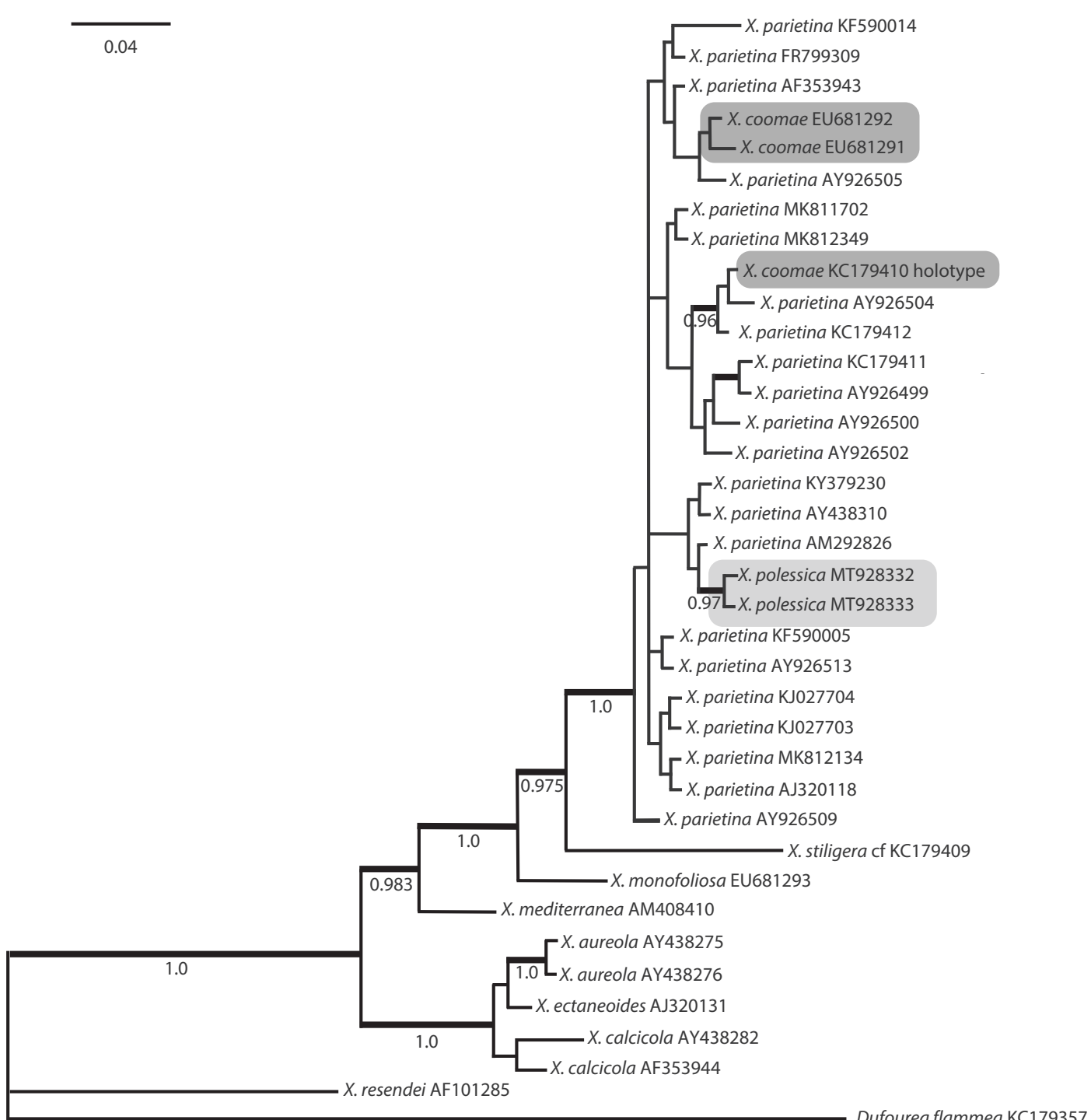

Figure 1. A majority-rule consensus tree based on an analysis of 36 ITS Xanthoria sequences using Bayesian MCMC. Dufourea flammea was used to root the tree. The tree shows Xanthoria coomae and X. polessica (highlighted in grey) nested within the X. parietina clade. Numbers below internodes indicate PP values $\geq 0.95$.

(represented by 22 sequences) including $X$. polessica (represented by two sequences from the type locality) and $X$. coomae (represented by three sequences including the holotype) nested in this highly supported clade. X. coomae appeared polyphyletically divided into two clades, with the type material clustered with $X$. parietina sequences from Norway and Chile $(\mathrm{PP}=0.96)$. The remaining two sequences of $X$. coomae form a weakly-supported clade with $X$. parietina sequences from Norway. The two identical sequences of $X$. polessica cluster with two sequences of $X$. parietina from Russia and Sweden, and an additional sequence of unknown origin.

\section{Discussion}

Lindblom \& Ekman (2005) investigated the phylogenetic relationship of Xanthoria parietina to $X$. aureola and $X$. calcicola using sequences of two molecular markers, one of which was the ITS. A total of 15 haplotypes differing mostly by a single point mutation were recognized within $X$. parietina. We have partially used sequences from their data set, but also included sequences from additional specimens representing a much wider geographical range. We observed a similar, low level of genetic variation within the ITS region of $X$. parietina including $X$. polessica and $X$. coomae, ranging from identical sequences to a single or rarely a few nucleotide difference among the specimens from different continents.

Based on morphology, X. polessica and X. coomae represent small forms of $X$. parietina differing in a few additional characters. $X$. polessica has a thicker thallus, smaller thalline lobes which are well-developed only in the peripheral zone, apothecia developing mainly in the central part of the thallus, which are surrounded by thick thalline margins, and smaller ascospores with a narrower ascospore septum (Kondratyuk et al. 2013). The fresh specimens collected from the type locality perfectly match the morphological and anatomical characters given above. The vouchers have small, rosette-like thalli, mainly $1-1.5 \mathrm{~cm}$ in diameter, with lobes not exceeding $2 \mathrm{~mm}$ in width and length, seen in peripheral zone, thallus in section 240-450 $\mu \mathrm{m}$ thick, abundant apothecia in the 
central part, with thalline margin to $0.2 \mathrm{~mm}$ thick, and widely ellipsoid ascospores (10.0-)11.0-12.9(-15.0) $\times$ $(6.0-) 6.5-7.5(-10.0) \mu \mathrm{m}, \mathrm{l} / \mathrm{b}(1.1-) 1.4-1.8(-2.2), \mathrm{n}=23$, with narrow septum $(2.5-) 3.6-5.5(-8.0) \mu \mathrm{m}$ in width. $X$. coomae differs from $X$. parietina by its more horizontally orientated lobes, slightly shiny, wrinkled and uneven central portion of the thallus, much paler yellowish peripheral zone, and more ellipsoid ascospores although of similar size and width of septum (Kondratyuk et al. 2007).

The phylogenetic analyses of the ITS region do not support the recognition of $X$. polessica and $X$. coomae as two independent species outside of $X$. parietina. Their nested placement within a monophyletic $X$. parietina justifies their synonymification and indicates that the observed morphotypes of $X$. polessica and $X$. coomae should be considered as infraspecific variation within $X$. parietina.

Specimens examined. Belarus, Gomel region, Kalinkovichi district, Ozarichi village, on wooden fence, 6 Oct. 2018, P. Bely (MSKH, GSU, LD).

Xanthoria parietina (L.) Th. Fr., Lich. Arct.: 69. 1860.

= Xanthoria coomae S.Y. Kondr. \& Kärnefelt, Bibl. Lichenol. 96: 167. 2007, syn. nov.

Type: Australia, New South Wales, vicinity of Cooma township, on roadside Populus nigra and Pinus radiata trees, on introduced trees in Cooma Lions Park, 31 Jan. 2004, Kondratyuk 20494 (CANB - holotype!, MEL, PERTH, HO - isotypes).

= Xanthoria polessica S.Y. Kondr. \& A.P. Yatsyna, Acta Bot. Hung. 55(3-4): 355. 2013, syn. nov.

Type: Belarus, Gomel region, Kalinkovichsky district, Ozarichi village, on wooden fence, 16 May 1967, N.V. Gorbach (MSK-L 5686 - holotype!, MSK-L 5652 - isotype).

\section{Acknowledgements}

We cordially thank anonymous reviewers and editors, Adam Flakus (Kraków) and Jolanta Miądlikowska (Durham), for their valuable comments and suggestions, and Prof. Mark R. D. Seaward (Bradford) for helpful suggestions and the linguistic corrections.

\section{References}

Arup, U., Søchting, U. \& Frödén, P. 2013. A new taxonomy of the family Teloschistaceae. Nordic Journal of Botany 31: 16-83.

Arup, U., Vondrák, J. \& Halici, M. G. 2015. Parvoplaca nigroblastidiata, a new corticolous lichen (Teloschistaceae) in Europe, Turkey and Alaska. The Lichenologist 47: 379-385.

Darriba, D., Taboada, G. L., Doallo, R. \& Posada, D. 2012. jModelTest 2: more models, new heuristics and parallel computing. Nature Methods 9: 772.

Gardes, M. \& Bruns, T. D. 1993. ITS primers with enhanced specificity for basidiomycetes - application for the identification of mycorrhizae and rusts. Molecular Ecology 2: 113-118.

Guindon, S. \& Gascuel, O. 2003. A simple, fast and accurate method to estimate large phylogenies by maximum likelihood. Systematic Biology 52: 696-704.

Katoh, K. \& Standley, D. M. 2013. MAFFT Multiple Sequence Alignment Software Version 7: Improvements in Performance and Usability. Molecular Biology and Evolution 30: 772-780.

Katoh, K., Misawa, K., Kuma, K. \& Miyata, T. 2002. MAFFT: a novel method for rapid multiple sequence alignment based on fast Fourier transform. Nucleic Acid Research 30: 3059-3066.

Kondratyuk, S. Y., Kärnefelt, I., Elix, J. A. \& Thell, A. 2007. Contributions to the Teloschistaceae of Australia. Bibliotheca Lichenologica 96: $157-174$.

Kondratyuk, S., Yatsyna, A. P., Lőkös, L., Galanina, I., Haji Moniri, M. \& Hur, J.-S. 2013. Three new Xanthoria and Rusavskia species (Teloschistaceae, Ascomycota) from Europe. Acta Botanica Hungarica 55: 351-365.

Kondratyuk, S. Y., Upreti, D. K., Mishra, G. K., Nayaka, S., Ingle, K. K., Orlov, O. O., Kondratiuk, A. S., Lőkös, L., Farkas, E., Woo, J.-J. \& Hur, J.-S. 2020. New and noteworthy lichen-forming and lichenicolous fungi 10. Acta Botanica Hungarica 62: 69-108.

Lindblom, L. \& Ekman, S. 2005. Molecular evidence supports the distinction between Xanthoria parietina and X. aureola (Teloschistaceae, lichenized Ascomycota). Mycological Research 109: 187-199.

Ronquist, F., Teslenko, M., van der Mark, P., Ayres, D. L., Darling, A., Höhna, S., Larget, B., Liu, L., Suchard, M. A. \& Huelsenbeck, J. P. 2012. MrBayes 3.2: efficient Bayesian phylogenetic inference and model choice across a large model space. Systematic Biology 61: 539-542.

White, T. J., Bruns, T., Lee, S. \& Taylor J. 1990. Amplification and direct sequencing of fungal ribosomal RNA genes for phylogenetics. In: Innis, M. A., Gelfand, D. H., Sninsky, J. J. \& White, T. J. (eds). PCR protocols: a guide to methods and applications, pp. 315-322. Academic Press. 